that CTLA-4 is involved in the mechanism responsible for suppression by Tregs, at least in the LTT assay for certain antigenic molecules. Since CTLA-4 is important in Nispecific $\mathrm{T}$ cell proliferation, Treg-antigen presenting cell interaction could be a possible mechanism in our case. Although the number of cases is limited in this study, our method represents a modified LTT with improved sensitivity for $\mathrm{Ni}$, thus warranting further investigation.

Disclosure. Financial support: none. Conflict of interest: none.

${ }^{1}$ Department of Dermatology,
University of Occupational and
Environmental Health, Iseigaoka,
Yahatanishi-ku, Kitakyushu
${ }^{807-8555, \text { Japan }}$
${ }^{2}$ Department of Dermatology,
Kyoto University Graduate School
of Medicine, Kyoto, Japan
${ }^{3}$ Department of Immunobiology,
Institute of Development, Aging and
Cancer, Tohoku University Sendai,
Japan
${ }^{4}$ Department of Dermatology,
Hamamatsu University School of
Medicine, Hamamatsu, Japan
<k-sugita@ @med.uoeh-u.ac.jp>

Kazunari SUGITA ${ }^{1}$ Kenji KABASHIMA ${ }^{2}$ Yu SAWADA ${ }^{1}$ Sanehito HARUYAMA ${ }^{1}$ Manabu YOSHIOKA ${ }^{1}$ Tomoko MORI ${ }^{1}$ Miwa KOBAYASHI ${ }^{1}$ Kouetsu OGASAWARA ${ }^{3}$ Yoshiki TOKURA ${ }^{4}$

1. Merk HF. Lymphocyte transformation test as a diagnostic test in allergic contact dermatitis. Contact Dermatitis 2005; 53: 246.

2. Hosoki M, Bando $E$, Asaoka $K$ et al. Assessment of allergic hypersensitivity to dental materials. Biomed Mater Eng 2009; 19: 53-61.

3. Minang JT, Arestrom I, Troye-Blomberg $M$ et al. Nickel, cobalt, chromium, palladium and gold induce a mixed Th1-and Th2-type cytokine response in vitro in subjects with contact allergy to the respective metals. Clin Exp Immunol 2006; 146: 417-26.

4. Nouri-Aria KT. Foxp3 expressing regulatory T-cells in allergic disease. Adv Exp Med Biol 2009; 665: 180-94

5. Cavani A, Mei D, Guerra E et al. Patients with allergic contact dermatitis to nickel and nonallergic individuals display different nickelspecific $\mathrm{T}$ cell responses. Evidence for the presence of effector CD8+ and regulatory CD4+ T cells. J Invest Dermatol 1998; 111 : $621-8$

doi:10.1684/ejd.2012.1641

\section{Herpetiform pemphigus with anti-Dsg 1 and full-length BP180 autoantibodies}

Herpetiform pemphigus (HP) has been considered an unusual clinical variant of pemphigus. HP shows vesicles arranged on the periphery of annular erythemas in a manner similar to dermatitis herpetiformes. Histopathologically, HP is characterized by eosinophilic spongiosis in the epidermis without apparent acantholysis. Intercellular deposition of immunoglobulin $\mathrm{G}(\mathrm{IgG})$ is usually detected by direct immunofluorescence (DIF) [1]. Previous authors have identified autoantibodies against desmoglein 1 (Dsg 1) or $160 \mathrm{kD}$ pemphigus foliaceus (PF) antigen in the majority of cases. It has been proposed that autoantibodies in HP may recognize different epitopes from those of classical pemphigus [2]. We report a patient who was clinically diagnosed with HP and was revealed as having anti-Dsg 1 and full-length BP180 autoantibodies.

A 42-year-old woman visited our clinic in October, 2009. She presented pruritic eruptions on her trunk and limbs which had started 2 years prior to her visit. The eruption was characterized by a generalized, and partially annular, edematous erythema with tense or erosive vesicles and bullae of variable sizes on the periphery (figure 1A). No abnormal lesion was found on her oral mucosa. Histopathology of a biopsy specimen taken from the vesicular lesion showed intraepidermal blistering containing eosinophils and neutrophils. In the epidermis, both acantholysis and eosinophilic spongiosis were also observed (figure 1B).

DIF revealed IgG deposition in the intercellular spaces (ICS) in the epidermis (figure 1C), but not in the basement membrane zone (BMZ). Under indirect immunofluorescence (IIF) with the patient's sera using normal human skin, the titer of anti-ICS IgG autoantibodies was found to be over 1:160, and that of anti-BMZ IgG autoantibodies to be $1: 10$. IIF using $1 \mathrm{M} \mathrm{NaCl}$-split human skin (ss-IIF) showed IgG antibodies in the epidermal side of the blister. Immunoblotting (IB) with epidermal extract showed reactivity against Dsg 1, full-length BP180, but no reactivity against Dsg 3, BP230, envoplakin, or periplakin (figure 1D).

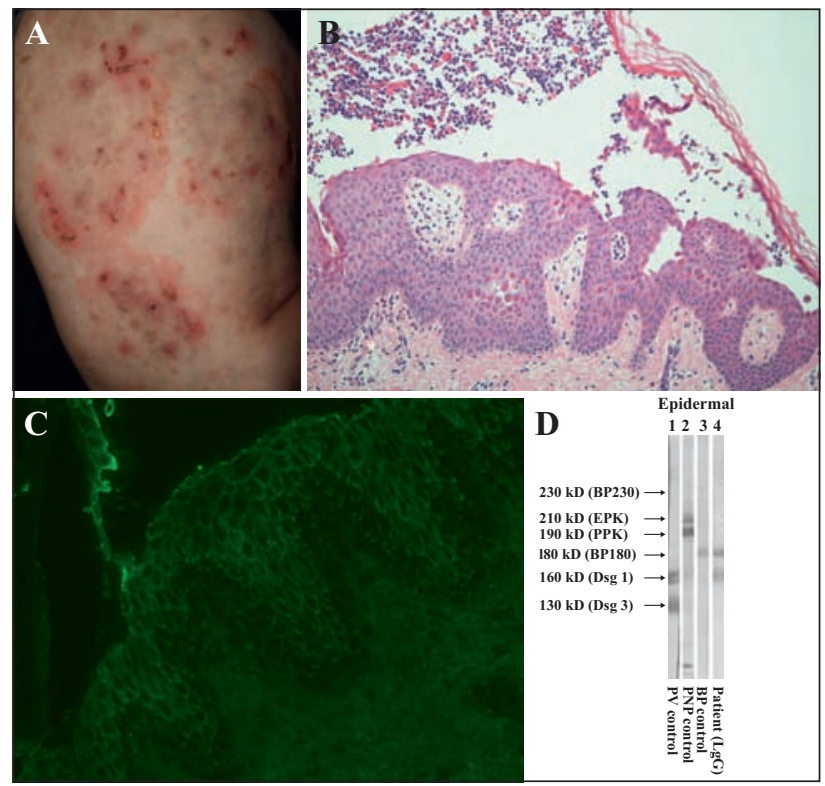

Figure 1. A) Clinical appearances on the femur. Annular, edematous erythema with vesicles on its periphery is shown. B) Histopathology revealed intraepidermal blisters and eosinophilic spongiosis. (hematoxylin-eosin, original magnifications: $\times 100)$. C) DIF revealed IgG deposition in the ICS of the epidermis. D) Reactivity of serum with human epidermal extracts by immunoblot procedures. The representative sera from pemphigus vulgaris (PV), paraneoplastic pemphigus (PNP), bullous pemphigoid (BP) are shown in lane 1-3. The patient serum (lane 4 ) shows reactivity against Dsg 1 and full-length BP180. 
IB showed no reactivity against the recombinant protein of NC16A or the C-terminal region of BP180. Enzyme-linked immunosorbent assay (ELISA) showed the titer of anti-Dsg 1 autoantibodies at 1:1620, anti-BP180 (NC16A) antibodies at 1:14 (positive level=more than 1:9), and no anti-Dsg-3 autoantibodies.

With the characteristic clinical picture, we diagnosed the patient with HP. Treatment with oral prednisolone starting at a daily dose of $60 \mathrm{mg}$ and gradually tapered to $25 \mathrm{mg}$, the condition was temporarily improved. Nevertheless, after leaving hospital, she rapidly reduced the prednisolone to $5 \mathrm{mg}$ of her own accord. After that, the eruption recurred immediately. After re-administration from $25 \mathrm{mg}$, with strict instructions, the condition has been well controlled. There have been some cases presenting immunological findings of both pemphigus and pemphigoid in the same patient $[3,4]$, in which the authors suggested that the additional "secondary" autoantibodies might be derived from the primary autoimmune bullous disease. This phenomenon was named epitope spreading (ES). ES is the sequential development of new antibodies against less accessible regions of target autoantigens in autoimmunity [5]. Maeda et al. [4] emphasized that simultaneous detection of autoantibodies in a single patient did not necessarily represent the concomitance of two different diseases, but rather that this fact could point to ES. On this basis, we regard the BP-like immunological findings presented in our case as an instance of ES.

Tchernev et al. [6] stated that, in cases of pemphigus, the formation of new antibodies may induce a non-typical clinical picture and may result in early recurrence if therapy is not maintained. Therefore, in our case, it is possible that atypical immunological findings, possibly interpreted as ES, may have contributed to establishing the characteristic clinical features and tendency for early recurrence.

Disclosure. Financial support: none. Conflict of interest: none.
${ }^{1}$ Department of Dermatology,

Tokyo Medical University, 6-7-1 Nishishinjuku Shinjuku-ku, Tokyo 160-0023, Japan

${ }^{2}$ Department of Dermatology, Kurume University, Kurume, Japan <kurasige@tokyo-med.ac.jp>

\section{Yuta KURASHIGE ${ }^{1}$ Yoshihiko \\ MITSUHASHI ${ }^{1}$ \\ Masuyoshi SAITO $^{1}$ \\ Shunpei FUKUDA ${ }^{2}$ \\ Takashi HASHIMOTO ${ }^{2}$ Ryoji TSUBOI $^{1}$}

1. Robinson ND, Hashimoto T, Amagai $M$ et al. The new pemphigus variants. J Am Acad Dermatol 1999; 40: 649-671; quiz 72-73.

2. Kubo $A$, Amagai $M$, Hashimoto $T$ et al. Herpetiform pemphigus showing reactivity with pemphigus vulgaris antigen (desmoglein 3 ). $\mathrm{Br}$ J Dermatol 1997; 137: 109-113.

3. Takahashi $T$, Anzai $H$, Suzuki $Y$ et al. Parallel fluctuation of antidesmoglein 3 and anti-BP 180 autoantibody titres in a patient of bullous pemphigoid. Clin Exp Dermatol 2004; 29: 608-611.

4. Maeda JY, Moura AK, Maruta CW et al. Changes in the autoimmune blistering response: $A$ clinical and immunopathological shift from pemphigus foliaceus to bullous pemphigoid. Clin Exp Dermatol 2006; 31: 653-655.
5. Hashimoto T, Hamada T, Dainichi T et al. How does intramolecular epitope spreading occur in BPAG2 (BP180)? J Invest Dermatol 2010; 130: 924-926.

6. Tchernev G, Orfanos CE. Antigen mimicry, epitope spreading and the pathogenesis of pemphigus. Tissue Antigns 2006; 68: 280-286.

doi:10.1684/ejd.2012.1643

\section{Does treatment of metabolic syndrome components improve psoriasis? Report of three cases.}

Psoriasis is a chronic, immune-mediated, inflammatory skin disease with a prevalence of $2-3 \%$. There is increasing awareness that psoriasis is more than "skin deep", with important systemic manifestations due to systemic inflammation with increased levels of inflammatory cytokines. Of emerging importance is the relationship between cardiovascular disease and severe psoriasis, explaining the increased mortality observed in psoriatic patients [1]. Recently, a strong association between increased adiposity, obesity and psoriasis has emerged. Several studies have shown a significant association between increased BMI and psoriasis and a recent prospective cohort study has confirmed that obesity precedes psoriasis, with body weight being directly associated with the risk for developing psoriasis. Furthermore, it has been shown that weight loss increases the responsiveness of psoriatic obese patients to low doses of cyclosporine [2]. In addition to obesity, psoriasis patients are more likely to have metabolic syndrome [2,3] which comprises three out of the following five criteria: abdominal obesity, hypertension, hypertriglyceridemia, low HDL cholesterol, and insulin resistance. Metabolic syndrome is currently considered a strong predictor of cardiovascular disease with a twofold rise in cardiovascular events and 1.5 fold increase in overall mortality.

We report 3 cases of patients with mild plaque-type psoriasis, with no psoriatic arthritis, and metabolic syndrome that improved after controlling the risk factors associated with metabolic syndrome, in the absence of any systemic or topical psoriatic drugs. All patients had lesions localized mainly on the extremities and trunk, with a mean PASI score of 6.1, and metabolic syndrome with diabetes mellitus type 2, hypertension, hypercholesterolemia, hypertriglyceridemia and obesity $(\mathrm{BMI}>30)$, that was under treatment but without good control. All patients started a strict diet, dyslipidemia was treated with atorvastatin, hypertension was controlled with increase dosage of the hypertensive drug and glycemic control was achieved with increased dosages of metformin and/or the addiction of glibenclamide. No systemic or topical psoriatic drug was used, except emollient. Demographic, clinical and therapeutic data are shown in table 1. After 6 months of diet and the new pharmacological treatment, an improvement of psoriasis severity of at least $50 \%$ was observed, along with significant loss of weight and BMI (from obesity to overweight), control of dyslipidemia, hypertension and glycemic control (table 1). 